Nuno Domingos, «Les reconfigurations de la mémoire du colonialisme portugais: récit et esthétisation de l'histoire», Histoire@Politique, [en ligne], n² 29, mai-août 2016, www.histoire-politique.fr

\title{
Les reconfigurations de la mémoire du colonialisme portugais : récit et esthétisation de l’histoire
}

\author{
Nuno Domingos
}

Dans l'un des premiers ouvrages sur le système colonial portugais publiés après le 25 avril 1974, Valentim Alexandre a mis en évidence combien les études sur le colonialisme pendant l'Estado Novo (l'État nouveau) ont mis en avant tout un ensemble de mythes nationalistes ${ }^{1}$. Parmi ces mythes, on trouve la théorie selon laquelle les Portugais auraient été les premiers à abolir la traite négrière. Un autre mythe porte sur la prétendue tendance culturelle des Portugais à créer des sociétés multiraciales, discours que l'État s'est approprié, dans les années 1950, en reprenant les travaux du sociologue brésilien Gilberto Freyre². À la même époque, l'idée de l'adaptabilité et de la plasticité des Portugais était présente dans des essais sur l'identité nationale, comme par exemple dans le travail de l'anthropologue J orge Dias $^{3}$. Dans le contexte de la lutte contre le colonialisme portugais, certains chercheurs avaient déjà dénoncé les contradictions entre le discours officiel promu par l'État depuis les années 1950 - et qui se distinguait des perspectives antérieures, principalement racialistes - et la réalité sur le terrain colonial ${ }^{4}$. Au début des années 1960, Charles Boxer remettait en question, de façon plus systématique, la propagande portugaise sur les relations raciales au sein de l'empire portugais ${ }^{5}$.

Si la fin du pouvoir colonial portugais en Afrique, avec la chute de l'Estado Novo le 25 avril 1974, a entraîné une modification des termes de la reproduction de la mémoire impériale, une certaine représentation du passé, une pastorale, pour utiliser l'expression de Raymond Williams, a continué à se reproduire, répondant à différents desseins institutionnels, à de nouvelles rhétoriques et profitant de nombreux silences ${ }^{6}$. Le développement d'études sur l'empire portugais n'a pas réussi à créer les

\footnotetext{
1 Valentim Alexandre, Origens do Colonialismo Português Moderno, Lisboa, Sá da Costa, 1979.

2 Gilberto Freyre, O Mundo que o Português Criou, Lisboa, Livros do Brasil, 1951; Gilberto Freyre, Integração Portuguesa nos Trópicos, Lisboa, Junta de Investigações do Ultramar, 1958 ; Gilberto Freyre, O Luso e o Trópico, Lisboa, Comissão Executiva das Comemorações do Quinto Centenário do Infante D. Henrique, 1961. Sur la réception de l'œuvre de Gilberto Freyre au Portugal, voir Cláudia Castelo, O Modo Português de Estar no Mundo. O luso-tropicalismo e a ideologica colonial portuguesa (1933-1961), Porto, Afrontamento, 2001; Cláudia Castelo, Marcos Cardão (dir.), Gilberto Freyre. Novas leituras do outro lado do Atlântico, São Paulo, Edusp, 2015.

${ }^{3}$ J orge Dias, Elementos fundamentais da cultura portuguesa, Coimbra, Tipografia Atlântica, 1955. Sur J orge Dias, voir Harry G. West, «Invertendo a Bossa do Camelo. J orge Dias, a sua Mulher, o seu Intérprete e eu », dans Manuela Ribeiro Sanches (dir.), Portugal não é um País Pequeno, Lisboa, Cotovia, 2005, pp. 141-190.

4 Marvin Harris, Portugal's African "wards": A First-hand Report On labor and Education in Moçambique, American Committee on Africa, 1958.

5 Charles Boxer, Race Relations in the Portuguese Colonial Empire, 1415-1825, Oxford, Clarendon Press, 1963.

${ }^{6}$ Raymond Williams, The Country and the City, New York, Oxford, University Press, 1973.
} 
Nuno Domingos, «Les reconfigurations de la mémoire du colonialisme portugais: récit et esthétisation de l'histoire », Histoire@Politique, [en ligne], n² 29, mai-août 2016, www.histoire-politique.fr

conditions appropriées pour questionner cette mémoire officielle, dans le champ universitaire, et encore moins au sein d'un espace public élargi, bien que des propositions pour penser cette expérience soient apparues dans la littérature, dans les arts plastiques, dans le cinéma de fiction et le documentaire, et même dans certaines émissions de télévision?

Il est plus difficile de saisir la subjectivation de la question impériale, de comprendre de quelle manière elle pénètre la mémoire collective, si elle est ou non présente dans les processus d'identification individuelle. Comparant la formation de la fierté nationale dans divers pays européens, l'International Survey Programme (Programme international d'enquêtes sociales) de 2003, avec les limites inhérentes à ce type de représentation statistique, révèle que la fierté des Portugais provient majoritairement de l'histoire de leur pays (91,9\% des réponses) ${ }^{8}$. D'autres indicateurs qui caractérisent typiquement le patriotisme civique présentent des résultats moins impressionnants : seulement 18,9\% des sondés sont fiers du système de sécurité sociale et 38,7\% du fonctionnement de la démocratie ${ }^{9}$. Si l'on prend en compte la place de l'empire dans la production de l'identité nationale, ces résultats suggèrent la propagation d'un récit héroïque de l'histoire du Portugal, récit qui a survécu à la fin de la dictature de l’Estado Novo.

La reproduction d'une certaine biographie d'Eusébio da Silva Ferreira, joueur de football né au Mozambique et figure majeure de la culture populaire portugaise, à la suite de son décès en janvier 2014, suscite un ensemble de questions sur la manière dont la mémoire coloniale s'est configurée.

L'entrée du joueur au Panthéon national, monument où reposent certains des plus illustres représentants de la nation portugaise, a clos le processus d'officialisation de la mémoire du joueur. L'institutionnalisation inédite d'un joueur de football s'est faite au nom d'un extraordinaire talent sportif qui a servi le pays à une période assez particulière, lorsque le Portugal combattait sur trois fronts afin de conserver son empire en Afrique. Dans le cas d'Eusébio, les récits issus d'un champ d'activité spécifique, largement médiatisé, comme le champ sportif, offrent un moyen plus adéquat de raconter le passé national pour construire une mémoire impériale d'une nation post-coloniale ${ }^{10}$. Transformé en héros national, le héros sportif permet un

\footnotetext{
${ }^{7}$ Sans viser l'exhaustivité, nous pouvons distinguer les récents livres d'Isabela Figueiredo (Caderno de Memórias Coloniais), de Dulce Maria Cardoso (O Retorno), le travail de plasticiens comme Ângela Ferreira et Vasco Araújo, les documentaires de Diana Andringa et la série télévisée de J oaquim Furtado sur la guerre coloniale. A la télévision également, il est nécessaire de faire référence au travail critique du comique Herman José, notamment dans ses parodies d'entretiens historiques avec de "grands hommes » (comme Luís Vaz de Camões, Afonso de Albuquerque et Dom Sebastião par exemple).

${ }^{8}$ J osé Manuel Sobral, « Dimensões étnicas e cívicas e glorificação do passado em representações da identidade nacional portuguesa numa perspectiva comparada», in J osé Manuel Sobral, Jorge Vala (eds), Identidade Nacional, inclusão e exclusão social, Lisboa, Imprensa de Ciências Sociais, 2010, p. 98. À titre de comparaison, notons que, dans une liste de 35 pays, seuls deux pays possèdent un « orgueil historique » plus élevé : les États-Unis et le Venezuela.

9 J oão Leal, "Ser português: um orgulho relativo», dans José Manuel Sobral, Jorge Vala (eds.), Identidade Nacional..., op.cit., p. 76. J uste après l'histoire apparaît la fierté dans les résultats sportifs (86,5 \%) et la qualité des artistes et des écrivains (84,8 \%).

10 Un champ d'activité peut être décrit « comme un réseau ou une configuration de relations objectives entre des positions. Ces positions sont définies objectivement dans leur existence et dans les déterminations qu'elles imposent à leurs occupants, agents ou institutions, par leur situation (situs) actuelle et potentielle dans la structure de la distribution des différentes espèces de pouvoir (ou de capital) dont la possession commande l'accès aux profits spécifiques qui sont en jeu dans le champ, et du même coup par leurs relations objectives aux autres positions (domination, subordination, homologies,
} 
Nuno Domingos, «Les reconfigurations de la mémoire du colonialisme portugais: récit et esthétisation de l'histoire », Histoire@Politique, [en ligne], n² 29, mai-août 2016, www.histoire-politique.fr

retour vers le passé, moins intrusif, qui s'articule mieux avec l'intention de l'État de nouer le passé impérial à la mémoire de la nation.

Certaines des dynamiques présentes dans l'appropriation d'Eusébio sont perceptibles dans d'autres situations contemporaines. L'analyse des legs de l'architecture coloniale moderne, dans le contexte des études de plus en plus nombreuses sur le patrimoine portugais dans le monde, offre un exemple de la manière dont le langage et l'histoire d'un autre champ d'activité spécifique - l'architecture - deviennent des véhicules de la narration du passé impérial. Cette patrimonialisation de l'histoire montre comment l'identité impériale est transmise par le commerce. Avec ces deux exemples, qui concernent deux champs d'activité distincts, le premier inscrit depuis longtemps dans l'imaginaire populaire et le second possédant un profil plus spécialisé et érudit, nous prétendons montrer comment une histoire coloniale portugaise apologétique s'est naturalisée. Néanmoins, ces images et ces représentations ne correspondent pas seulement à ce qui, dans l'idéal, pourrait être décrit comme l'intérêt des grands acteurs historiques, en premier lieu l'État et le champ politique et bureaucratique. Elles s'inscrivent également dans l'histoire et la généalogie de champs d'activité spécifiques, champs qui confèrent une base institutionnelle et des mécanismes de médiation qui rendent la subjectivation d'une mémoire coloniale bien plus efficace et subtile. Ces champs offrent des dispositifs de narration qui permettent de raconter, à partir de rhétoriques distinctes, cette période si marquante de l'histoire portugaise et de la construction de l'identité portugaise. Ces brèves études de cas suggèrent également que les langages des champs spécifiques ont un pouvoir plus large d'euphémisation des principaux éléments d'un discours du pouvoir, contribuant au renforcement et à légitimité de sa reconnaissance. Ces médiations sont fondamentales pour l'interprétation des logiques de reproduction d'une mémoire coloniale depuis la fin de l'empire.

\section{L'empire comme axe de production d'une identité nationale}

L'histoire de l'empire a été essentielle dans la production de l'identité nationale portugaise. L'interprétation du rôle de l'empire dans ce processus prolonge la discussion sur les origines et les dynamiques d'une identité portugaise, l'origine et la chronologie du sentiment patriotique et national et le rôle de l'État et $d u$ nationalisme dans la formation de cette identité11.

etc. », Pierre Bourdieu, Loïc Wacquant, Réponses. Pour une anthropologie réflexive, Paris, Seuil, 1992, pp. $72-73$.

${ }^{11}$ Parmi les ouvrages qui ont discuté cette question depuis le 25 avril 1974, voir Martim de Albuquerque, A Consciência Nacional Portuguesa, Lisboa, Edição do Autor, 1974 ; J osé Mattoso, Identificação de um País. Ensaio sobre as Origens de Portugal, 2 vol., Lisboa, Editorial Estampa, 1985; Francisco Bethencourt, Diogo Ramada Curto (eds.), A Memória da Nação, Lisboa, Livraria Sá da Costa, 1991; J oão Leal, Etnografias Portuguesas (1870-1970): Cultura Popular e Identidade Nacional, Lisboa, Publicações Dom Quixote, 2000 ; Vitorino Magalhães Godinho, Portugal : a Emergência de uma Nação, Lisboa, Colibri, 2004 ; Sérgio Campos Matos, Consciência Histórica e Nacionalismo - Portugal, séculos XIX e XX, Lisboa, Livros Horizonte, 2008 ; José Neves, Comunismo e Nacionalismo em Portugal - Política, Cultura e História no Século XX, Lisboa, Tinta da China, 2008 ; Luís Trindade, O Estranho Caso do Nacionalismo Português, Lisboa, Imprensa de Ciências Sociais, 2008 ; Patrícia Ferraz de Matos, As Cores do Império: Representações Raciais no Império Colonial Português, Lisboa, Imprensa de Ciências Sociais, 2006 ; José Manuel Sobral, Portugal, Portugueses: uma identidade 
Nuno Domingos, «Les reconfigurations de la mémoire du colonialisme portugais: récit et esthétisation de l'histoire », Histoire@Politique, [en ligne], n² 29, mai-août 2016, www.histoire-politique.fr

La célébration de l'empire a accompagné la constitution d'un champ politique en évolution et le développement d'un État moderne, principal producteur symbolique de la grandeur nationale. La transmission d'un ensemble de références sur l'empire découle également d'initiatives réalisées par des institutions comme l'Église catholique et des processus de socialisation inhérents aux cercles de relations familiales et de proximité12. Mais l'État, notamment à l'époque contemporaine, assume l'avant-garde de la nationalisation des masses.

Moyen de socialisation identitaire, l'empire s'est transformé en une langue de communication avec la population déjà initiée aux choses coloniales, notamment depuis l'utilisation, par les régimes libéraux, à partir des années 1820, de la nation comme instrument symbolique de l'unité du territoire et de la population ${ }^{13}$. Après l'ultimatum anglais de 1890, lorsque le gouvernement britannique s'est opposé aux velléités du Portugal d'occuper des territoires entre l'Angola et le Mozambique, la fierté nationale s'est confondue avec la fierté impériale. Cette identification a perduré au cours de l'ascension du mouvement républicain, qui a conduit à la chute de la monarchie en 1910, puis tout au long de la première République et pendant l'Estado Novo. La légitimité de l'empire a été tardivement partagée par les opposants à l'Estado Novo, alors même qu'ils critiquaient les méthodes de l'administration coloniale ${ }^{14}$. Cette identification ne dépendait pas seulement des convictions profondes des dirigeants socialisés dans l'environnement national, ou de leurs intérêts plus ou moins déclarés : en se posant comme moyen de communication avec la population, l'empire s'est converti en capital politique. Ce nationalisme impérial a commencé à être contesté après la Seconde Guerre mondiale et, ensuite, de manière plus vigoureuse, dès le début du processus de décolonisation en Afrique. La guerre coloniale (1961-1974) a provoqué des résistances externes, des fractures importantes au sein des classes dominantes portugaises et une opposition interne plus diversifiée et ouvertement anti-colonialiste ${ }^{15}$. Le consensus concernant la place de l'empire dans la construction de l'identité nationale s'est rompu, de manière décisive, à la suite du 25 avril 1974. En dépit des luttes pendant la période révolutionnaire, les forces qui défendaient l'indépendance des colonies africaines ont obtenu une hégémonie au sein de l'appareil étatique, dans le champ politique et culturel ${ }^{16}$. Toutefois, la position désormais dominante qui défendait la décolonisation rapide a rencontré des résistances. Certains mouvements ont revendiqué le maintien de liens entre le Portugal et ses anciennes colonies ou d'autres modalités de décolonisation. Une grande partie des colons qui quittèrent les colonies en 1974 et en 1975 en direction de la métropole adhérait à ces thèses continuant de défendre l'empire ${ }^{17}$. Les partis les

nacional, Lisboa, FFMS, 2014 ; J osé Manuel Sobral, J orge Vala (eds), Identidade Nacional, inclusão e exclusão social, Lisboa, Imprensa de Ciências Sociais, 2010.

12J osé Manuel Sobral, Portugal, Portugueses..., op. cit., p. 88.

13 Sérgio Campos de Matos, « Nação », Ler História, n 55, 2008, p. 111-124. « Le mot "Nation" acquiert une place centrale dans le discours politique du premier libéralisme - il est présent lors du serment par le Roi des bases de la Constitution, dans les Cortes (1821) et dans le serment des députés. Le terme Royaume, lui, perd de son importance» (p.117). La nation comme concept clé de la politique du libéralisme est un concept importé : « Les libéraux ont progressivement assimilé Nation et État, récusant la souveraineté du Roi » (p. 120).

14 J osé Neves, Comunismo e Nacionalismo..., op. cit., p. 135-144.

15 Miguel Cardina, Margem de Certa Maneira. O Maoísmo em Portugal 1964-1974, Lisboa, Tinta da China, 2011.

${ }^{16}$ António Costa Pinto, O Fim do Império Português, Lisboa, Livros Horizonte, 2001, p. 80.

17 Ibid., p. 83. 
Nuno Domingos, «Les reconfigurations de la mémoire du colonialisme portugais: récit et esthétisation de l'histoire », Histoire@Politique, [en ligne], n² 29, mai-août 2016, www.histoire-politique.fr

plus à droite du champ politique portugais se sont érigés en représentants des perdants de la chute de l'empire, bien que leur discours ne prétendît pas récupérer la souveraineté de l'ancien espace impérial. Ainsi, les projets de récupération de l'autorité impériale n'avaient aucune représentation politique, à l'exception de petits groupes d'extrême droite ${ }^{18}$. Plusieurs sondages réalisés après le 25 avril 1974, à différents moments, suggéraient que la plupart des Portugais, même s'ils n'étaient pas d'accords avec les modalités de la décolonisation, la trouvaient juste19.

Après 1974, la représentation de la nation portugaise a été concurrencée par d'autres imaginaires proposés au sein du champ politique. Les projets d'un Portugal socialiste et révolutionnaire ou d'un Portugal européen stabilisé dans le cadre d'une démocratie libérale, comme cela est advenu à partir de 1976, ont mis de côté l'imaginaire impérial. L'empire s'est dissout en tant que moyen de production de la grandeur nationale. Les guerres civiles qui ont divisé plusieurs anciennes colonies portugaises, notamment en Angola et au Mozambique, se sont alors réalisées dans le contexte géopolitique de la guerre froide.

Sous couvert du discours de la diplomatie nationale, des relations économiques et institutionnelles se sont nouées, d'anciens réseaux se sont maintenus et de nouvelles opportunités se sont créées. La diplomatie, renforcée par l'entrée du Portugal dans la Communauté économique européenne en 1986, a offert à l'État portugais un statut d'intermédiaire entre l'Europe et des zones d'influence en Afrique, en Amérique latine et en Asie. En 1996, la création de la Comunidade de Países de Língua Portuguesa (Communauté de pays de langue portugaise, CPLP), justifiée par un discours de partage historique et de l'échange culturel, proposait une imaginaire lusophone. Stratégie de négociation de la mémoire de l'empire, la lusophonie a offert une vision particulière des relations entre les espaces de langue portugaise, et incitait à la reconnaissance de ce qui était positif et acceptable, au nom d'une relation institutionnelle favorable à la diplomatie et aux affaires. Si l'exaltation souverainiste a disparu, des allusions plus neutres à ce passé se sont imposées, plus suggestives que prescriptives, parfois protégées par l'euphémisme, mais surtout basées sur le silence concernant le passé.

Mais l'histoire de l'empire ne pouvait pas simplement disparaître des processus liés à l'imagination nationale. Au final, ce passé n'était pas seulement reproduit dans le champ politique : il était sédimenté dans les mémoires individuelles et familiales. Il s'était également imposé comme une dimension fondamentale de l'histoire d'institutions directement impliquées dans la conquête coloniale, comme l'institution militaire et religieuse, notamment par l'intermédiaire de l'action missionnaire de

\footnotetext{
18 Riccardo Marchi, «A identidade de Portugal no discurso da direita radical : do multirracialismo ao etnonacionalismo », Estudos Ibero-Americanos, 41(2), 2015, pp. 422-442.

19 Selon l'étude de Mário Bacalhau, citée par António Costa Pinto, en 1978, 70 \% des Portugais considéraient que l'indépendance devait être donnée aux colonies, tout en sauvegardant les droits des Portugais installés là-bas. Seulement 2,2\% considéraient que la guerre aurait dû continuer. Francisco Bethencourt cite un sondage du quotidien O jornal, du 19 avril 1984, dans lequel $69 \%$ des Portugais sont s'accords avec la décolonisation et $22 \%$ opposés. Plus de $73 \%$ contestaient les modalités de la décolonisation. Francisco Bethencourt, «A Memória da Expansão », dans Francisco Bethencour, Kirti Chaudhuri (eds.), História da Expansão Portuguesa, vol. 5, Lisboa, Círculo de Leitores, 1999, p. 480. Plus récemment, une enquête sur le quarantième anniversaire du 25 avril semblait confirmer ces chiffres : $68 \%$ des réponses considéraient que la décolonisation était un sujet totalement ou assez résolu : Marina Costa Lobo, «As atitudes dos portugueses em relação aos 40 anos do 25 de Abril », Relatório para a Conferência ICS, 14 de Abril de 2014, Lisboa, Fundação Calouste Gulbenkian, 2014, p. 19.
} 
Nuno Domingos, «Les reconfigurations de la mémoire du colonialisme portugais: récit et esthétisation de l'histoire», Histoire@Politique, [en ligne], n² 29, mai-août 2016, www.histoire-politique.fr

l'Église catholique. Les mécanismes de consécration symbolique au sein de l'institution militaire impliquaient, par exemple, que les mêmes militaires qui étaient contre la guerre coloniale et le colonialisme portugais, reconnussent les valeurs, les devoirs et les hiérarchies inhérentes à la fonction militaire dans les colonies. Ainsi, le terrain colonial continuait à être représenté comme un lieu d'héroïsme, de camaraderie et d'esprit de corps. Les fréquentes rencontres d'anciens militaires construisent, de manière singulière, une représentation de l'empire comme un moment de consécration institutionnelle, basée sur de forts liens affectifs ${ }^{20}$. Pour l'Église catholique, l'exercice de la conversion des populations, efficace en d'innombrables parcelles de l'empire, constitue un moment de célébration, indépendamment de la discussion sur le rôle de l'Église dans les processus de domination coloniale ${ }^{21}$. Des processus semblables existent dans le champ scientifique. La conquête coloniale a promu le développement de différentes disciplines, la création d'institutions et la formation de spécialistes. Ce passé des différentes disciplines s'est transformé dans un espace spécifique dans lequel la mémoire coloniale est l'objet de controverses ${ }^{22}$.

Historiquement, les sphères de la vie sociale portugaise qui, d'une manière ou d'une autre, ont produit un discours sur l'expérience coloniale utilisent différents moyens pour propager leur point de vue.

\section{Comment raconter la nation}

Le processus de constitution de la mémoire impériale s'est construit sur plusieurs éléments : sur la fonction séculaire d'une culture écrite officielle comme moyen de raconter l'empire, progressivement appuyée par une iconographie, essentielle pour représenter l'empire dans un pays où une grande partie de la population était analphabète; sur la matérialisation de la mémoire impériale dans l'espace public ; sur les grandes cérémonies; et sur la production scientifique ${ }^{23}$. Plusieurs auteurs ont mis en exergue les statues et la toponymie impériales, tant dans l'espace métropolitain que dans l'empire même, la constitution d'une héraldique ultramarine, l'organisation de rituels et de cérémonies évocatrices dans les commémorations des

\footnotetext{
20 Elsa Peralta, «O Monumento aos Combatentes : A performance do fim do Império no espaço sagrado da nação », dans Paula Godinho (dir.), Antropologia e Performance, Castro Verde, 100 Luz, 2014.

${ }^{21}$ Miguel Bandeira J erónimo, A Diplomacia do Império. Política e Religião na Partilha de África (18201890), Lisboa, Edições 70, 2012.

22 Concernant les sciences sociales, voir Harry G. West, «Invertendo a Bossa..., op. cit. ; Rui Mateus Pereira, Conhecer para Dominar, O desenvolvimento do Conhecimento Antropológico na Política Colonial Portuguesa em Moçambique, 1926-1959, thèse de doctorat en anthropologie, Université Nouvelle de Lisbonne, 2005; Frederico Agoas, «Estado, Universidade e ciências sociais: um programma de pesquisa », dans Miguel Jerónimo (dir.), O Império Colonial em Questão, Lisboa, Ediçoes 70, 2012, p. 338-339.

23 Diogo Ramada Curto, «Língua e memória », dans J oaquim Romero Magalhães (dir.), Historia de Portugal, vol. 3 : No alvorecer da modernidade, 1480-1620, Lisboa, Circulo de Leitores, 1993, p. 357373 ; Maria Isabel J oão, « Comemorações e Mitos da Expansão », dans Francisco Bethencourt e Kirti Chaudhuri (eds.), História da Expansão Portuguesa, vol. 4, Lisboa, Círculo de Leitores, 1999, p. 403424 ; Maria Isabel J oão, Memória e Império. Comemorações em Portugal (1880-1960), Lisboa, Fundação Gulbenkian, 2002 ; Francisco Bethencourt, «A Memória da Expansão...», op. cit., p. 442480.
} 
Nuno Domingos, «Les reconfigurations de la mémoire du colonialisme portugais: récit et esthétisation de l'histoire », Histoire@Politique, [en ligne], n² 29, mai-août 2016, www.histoire-politique.fr

dates historiques ${ }^{24}$, l'organisation de grandes expositions, espaces de propagande mais aussi lieux de constitution d'une archive historique et idéologique ${ }^{25}$. Le système scolaire, par l'intermédiaire de cartes, de photographies, de dessins, d'histoires de héros nationaux, de campagnes militaires, d'espaces bâtis, de représentations souvent stéréotypées des populations subalternes, inculquait aux élèves portugais la croyance selon laquelle le Portugal n'était pas un petit pays mais un énorme empire². La constitution d'une historiographie de vulgarisation s'est appuyée en grande partie sur le recours au genre biographique, dont le potentiel dramatique et la structure linéaire ont facilité le récit d'une entité nationale27. Recourant à l'imaginaire, au mythologique, à l'utopique, à la légende et à la tradition populaire, on racontait des histoires de succès et on faisait l'éloge de conduites modèles ${ }^{28}$. Plus rigoureuse dans la méthode et dans l'utilisation des sources, l'historiographie érudite entrait en relation, de diverses manières avec cette historiographie de vulgarisation. L'autonomie de la science face au pouvoir politique était limitée et la nation infirme, décadente, en retard - se trouvait à l'origine de la formulation des questionnements scientifiques: les intérêts suprêmes de la nation et de l'empire, formule vague qui cachait la diversité des valeurs et des stratégies qui s'abritaient en son sein, ont été cruciaux pour le développement d'innombrables champs scientifiques au Portugal.

C'est à la frontière entre le besoin de connaître et de dominer et le besoin de créer des représentations de territoires et de populations justifiant la souveraineté politique et la supériorité civilisationnelle, en particulier dans le cadre de l'occupation effective de l'empire africain, que s'inscrit l'activité de plusieurs institutions créées à partir du dernier quart du XIX ${ }^{\mathrm{e}}$ siècle. Ce fut ainsi le cas de la Société de Géographie de Lisbonne (1875), de l'École coloniale (1906) - qui devient plus tard l'Institut supérieur de sciences politiques et sociales d'outre-mer - , de l'Agence générale des Colonies (1924), de la Junte de recherches de l'outre-mer ou du Centre d'études politiques et sociales. Les institutions scientifiques ont mené des recherches, ont produit et publié des documents. Elles ont organisé des archives - comme les Archives des colonies en 1917- et réalisé des séries statistiques et des

\footnotetext{
24 Notamment le 10 juin - jour de la race - , le 5 octobre (1910), date de l'implantation de la République, le 28 mai (1926) qui commémore, sous l’Estado Novo, la fin de la Première République, le 1er décembre (1640), date de la restauration et fin de la domination castillane.

25 Patrícia Ferraz de Matos, « Power and identity : the exhibition of human beings in the Portuguese great exhibitions », Identities : Global Studies in Culture and Power, n 21 (2) 2014, p. 202-218 ; Nadia Vargaftig, «L'Afrique des combattants : rêves et réalités à travers les expositions coloniales portugaises (1918-1940) », dans David Plouviez (dir.), Défense et colonies dans le monde atlantique, XVe-XXe siècle, Rennes, PUR, 2013.

26 J oão Carlos Paulo, « Da "educação colonial portuguesa" ao ensino no Ultramar », dans Francisco Bethencourt, Kirti Chaudhuri (eds), História da expansão portuguesa, vol. 5: Ultimo império e recentramento (1930-1998), Lisboa, Temas e Debates, 2000, p. 304-333. Sur une actualisation de ces lignes d’analyse voir Marta Araújo, Silvia Rodriguez Maeso, « Explorando o Eurocentrismo nos manuais portugueses de História », Estudos de Sociologia, n 15(28), 2010, p. 239-270.

27 Sérgio Campos de Matos, História, Mitologia..., op. cit.

28 Sérgio Campo de Matos, Historiografia e memória nacional. 1846-1898, Lisboa, Colibri, 1998. L'auteur énumère les genres de cette historiographie : histoires générales du Portugal, récits historiques liés à certains épisodes passés (à ne pas confondre avec le roman historique), biographies, certains feuilletons, livres scolaires, petits textes commémoratifs dans des revues ou des almanachs, pamphlets où prédomine l'argumentation historique.
} 
Nuno Domingos, «Les reconfigurations de la mémoire du colonialisme portugais: récit et esthétisation de l'histoire », Histoire@Politique, [en ligne], n² 29, mai-août 2016, www.histoire-politique.fr

recensements ${ }^{29}$. Soumise à des agendas économiques et politiques, l'exploration scientifique de l'empire, conduite avec plus ou moins de compétence, d'autonomie et de créativité, dépendait des techniques partagées dans un champ scientifique international en évolution, constitué par des institutions aussi bien étatiques, interétatiques que privées dans lesquelles le Portugal était représenté30.

Pendant l'Estado Novo, les organisations chargées de la propagande diffusaient ces connaissances par le biais de documents imprimés, de fictions cinématographiques, de documentaires et d'émissions de radio ${ }^{31}$. Cet imaginaire impérial a pénétré les circuits commerciaux, ajoutant une dimension importante à la socialisation impériale ${ }^{32}$. Récemment, l'historien Marcos Cardão a montré comment la représentation luso-tropicale du monde portugais, opérée par l'appropriation politique des travaux de Gilberto Freyre, s'est diffusée dans les années 1950 et 1960 par le biais de la musique, du football et des concours de beauté33. Loin d'être de simples épiphénomènes de l'idéologie nationale ou étatique, les champs culturels, scrutés durant l'Estado Novo par une censure qui empêchait tout discours dissonant, ont été des moyens de socialisation d'une idéologie nationale et impériale.

Il est possible d'évaluer aujourd'hui la sédimentation d'un imaginaire impérial dans la circulation d'une littérature commerciale, dans des programmes télévisés à large audience et dans l'industrie du tourisme ${ }^{34}$. Elément d'identification des Portugais en tant que Portugais, la mémoire de l'empire a créé les conditions pratiques et symboliques pour l'existence d'un tel marché. Le désir d'utiliser l'histoire et le patrimoine, qui constituent des ressources qui ajoutent de la plus-value aux marchandises et aux services, possède actuellement une dimension significative dans la reproduction de cette histoire exemplaire. Face à l'impossibilité de maintenir un discours ouvertement nostalgique sur l'empire au sein du champ du pouvoir postcolonial, le développement de nouvelles pratiques et de consommations culturelles populaires qui se sont développées grâce aux moyens de communication et à l'alphabétisation a offert de nouvelles opportunités de médiation à la construction nationale. L'histoire d'une pratique populaire comme le football crée les conditions d'un récit transmettant un ensemble de représentations sur le quotidien. Lors de nombreux moments historiques, ces représentations ont été utilisées pour renforcer les principes de division du monde à la base de la constitution d'un champ du pouvoir. Le cas du joueur de football Eusébio da Silva Ferreira, héros de la culture populaire, est particulièrement opérante pour raconter l'histoire idéalisée du pays.

\footnotetext{
29 Agrégées dans des publications comme l'Annuaire Statistique de l’Outre-Mer, de 1943, ou découlant du travail des instituts de statistiques de chaque colonie.

30 Le même processus se trouve dans d'autres contextes coloniaux, voir Frederick Cooper, Randall Packard (eds.), International Development and the Social Sciences, Berkeley, University of California Press, 1997. Sur le Portugal, voir Rui Mateus Pereira, Conhecer para Dominar..., op. cit.

${ }^{31}$ Le Secrétariat de la Propagande nationale et son successeur, le Secrétariat national de l'Information, ainsi que l’Agence générale des Colonies (qui devient ensuite l'Agence générale de l’Outre-mer, de l'Information et du Tourisme), ont participé à une représentation idyllique de l'empire.

32 Luís Trindade défend que l'ascension du salazarisme, dans les années 1930, s'appuie substantiellement sur la profusion d'une littérature nationaliste dans les années précédentes: Luís Trindade, O Estranho caso..., op. cit.

33 Marcos Cardão, Fado Tropical. O luso-tropicalismo na cultura de massas, Lisboa, Edições Unipop, 2015.

34 Question qui intéressait déjà l’historien Vitorino Magalhães Godinho dans « O naufrágio da memória nacional e a Nação no horizonte do "marketing" », dans Francisco Bethencourt, Diogo Ramada Curto (eds.), A Memória da Nação..., op. cit.
} 
Nuno Domingos, «Les reconfigurations de la mémoire du colonialisme portugais: récit et esthétisation de l'histoire», Histoire@Politique, [en ligne], n² 29, mai-août 2016, www.histoire-politique.fr

\section{Eusébio da Silva Ferreira : le héros de la culture populaire}

Eusébio da Silva Ferreira, héros du club Sport Lisboa e Benfica et de la sélection nationale dans les années 1960, n'était pas prédisposé à rejoindre la famille des héros nationaux et de l'empire. Au bout du compte, il était seulement un extraordinaire joueur de football. Néanmoins, au cours du XX $\mathrm{X}^{\mathrm{e}}$ siècle, ce sport s'est affirmé comme une puissante ressource de représentation nationale. Faisant passer l'intérêt sportif après la dynamique nationaliste, les rencontres entre nations - particulièrement lors des grandes compétitions - acquièrent une valeur extra-sportive. Mais cette nation représentée par les artistes du ballon n'est pas une entité abstraite: elle est dotée d'un système politique, d'une structure sociale et économique, d'un régime et d'une d'idéologie ${ }^{35}$. Eusébio a été érigé en héros national dans les années 1960, au début de la lutte de l'Estado Novo pour conserver son empire en Angola (1961), en Guinée (1963) et au Mozambique (1964), à une époque où presque tous les pays africains avaient obtenu leur indépendance.

Eusébio ne doit pas son parcours à son seul talent. Né dans le bidonville de Mafalala, dans les banlieues pauvres et racialement ségrégées de Lourenço Marques, capitale du Mozambique, Eusébio se passionne pour un jeu pratiqué depuis le début du $\mathrm{XX}^{\mathrm{e}}$ siècle dans la périphérie de la ville ${ }^{36}$. Dans les années 1920, différents clubs de la périphérie se sont rassemblés au sein de l'Associação de Futebol Africana (AFA : Association africaine de football). Créée en 1924 en s'inspirant de l'associativisme sportif sud-africain, cette association réunissait seulement des joueurs noirs et métis. Ces joueurs noirs et métis avaient été écartés de l'Association de football de Lourenço Marques, créée en 1923 par des équipes de colons. Cette opposition entre les deux associations prend fin en 1959, quand l'État colonial portugais ferme l'AFA, pour éliminer l'un des vestiges institutionnels d'un racisme alors généralisé. À l'inverse d'autres joueurs qui n'ont jamais pu révéler leur talent hors de la banlieue, Eusébio a bénéficié de cette ouverture politique relative due à ce colonialisme tardif. Il est ainsi passé des matchs de banlieue au championnat du centre de la ville. À cette époque, le football connaissait un processus de professionnalisation rapide et la quête de bons athlètes transcendait les blocages raciaux. Certains transferts de joueurs africains vers le championnat de la métropole, comme ceux de Matateu ou de Mário Coluna, ont stimulé un marché impérial dont l'État portugais n'a autorisé l'existence qu'à partir de 1949. Provenant d'un environnement sportif déjà stabilisé, ces joueurs ont trouvé dans la métropole les conditions nécessaires pour développer leurs qualités. Et c'est grâce à ces nouveaux joueurs noirs et métis, que Benfica a gagné la Coupe des champions européens en 1961 et en 1962.

Mais c'est surtout la participation de la sélection nationale au Mondial de 1966 en Angleterre qui a donné tout son sens politique à la présence d'Africains dans l'équipe nationale. Leurs performances remarquables ont fait retentir le nom du Portugal à travers le monde. La glorification inédite d'un Africain noir au Portugal a octroyé un rôle émergent à la culture populaire dans la construction du nationalisme moderne

\footnotetext{
35 C'est un thème qui a été étudié dans divers contextes politiques, en Italie pendant le fascisme, en Argentine, en 1978, sous la dictature militaire, au Brésil en 1970, pendant la dictature militaire.

36 Nuno Domingos, Futebol e Colonialismo. Corpo e Cultura Popular em Moçambique, Lisboa, Imprensa de Ciências Sociais, 2012.
} 
Nuno Domingos, «Les reconfigurations de la mémoire du colonialisme portugais: récit et esthétisation de l'histoire», Histoire@Politique, [en ligne], n² 29, mai-août 2016, www.histoire-politique.fr

démontrant comment ces processus pouvaient être contradictoires ${ }^{37}$. Le pouvoir politique a saisi cette opportunité pour interpréter ce phénomène à son avantage. Faisant figure de rareté à l'époque parmi les sélections nationales européennes, l'équipe multiraciale portugaise pouvait représenter de manière efficace l'image d'un colonialisme exceptionnel, que l'Estado Novo défendait alors sur le terrain militaire ${ }^{38}$. Exploitée par la presse généraliste et spécialisée, la fierté footballistique est devenue un instrument idéologique subtil et efficace. Et Eusébio est devenu le grand protagoniste de cette représentation particulière de la patrie sur le terrain de football. Meilleur joueur et buteur de la Coupe du Monde de 1966, son visage et le maillot de la sélection nationale ont été reproduits par la presse internationale. On se demandait alors qui était le meilleur joueur de tous les temps : Eusébio ou le brésilien Pelé ?

Contrairement à la majorité des héros nationaux, Eusébio n'était pas une «production » typique de l'État-nation. À l'école, comme dans d'autres espaces institutionnels, la célébration de ce grand Portugal a fait appel au récit des vies exemplaires des héros nationaux. Le libéralisme a promu les héros du nouveau régime : des écrivains, des hommes d'État, des hommes politiques, les représentants d'une nouvelle nation civique. Mais, en dépit de l'avènement de ces hommes lettrés, la grandeur de la nation continuait à être racontée à travers les exemples paradigmatiques des militaires et des « explorateurs ${ }^{39}$ ». L'empire, en tant que lieu du héros national, connaît une renaissance avec la commémoration des centenaires ${ }^{40}$ : le quatrième centenaire de l'arrivée de Vasco de Gama en Inde en 1898, le troisième centenaire de la mort de Camões, le héros érudit qui raconte la grande histoire nationale, en 1880. On célèbre également les explorateurs en Afrique - Serpa Pinto (1846-1900), Hermenegildo Capelo (1841-1917) et Roberto Ivens (1850-1898) - , puis les chefs militaires des campagnes d'Afrique, comme Mouzinho de Albuquerque (1855-1902) et António Enes (1848-1901) ${ }^{41}$.

Eusébio doit sa notoriété à une activité populaire vulgarisée par la presse, moyen privilégié de diffusion des visions du monde. Bien qu'un match de football puisse être assimilé à un champ de bataille, Eusébio n'appartenait pas à la généalogie des grands hommes d'armes ou de lettres. Il ne ressemblait en rien aux héros de l'empire, aux navigateurs, aux militaires, aux gouverneurs, tous des hommes blancs. Mais son énorme popularité n'était pas négligeable. Il ne faisait déjà plus simplement partie de l'histoire du football. Sa promotion au rang de héros national, figure plus noble et légitime, exigeait une biographie appropriée, qui parle de ses faits sportifs, déjà largement connus, mais qui le construise aussi comme un homme. Cela permettait de raconter d'une manière plus convenable la nation et l'empire.

Cette biographie a commencé à être construite quelques années après l'arrivée d’Eusébio à Lisbonne, à la fin de 1961, alors qu'il débarquait de sa ville natale de Lourenço Marques. En 1966, la publication de son autobiographie, rédigée par

\footnotetext{
${ }^{37}$ Sur l’histoire des noirs au Portugal, voir J osé Ramos Tinhorão, Os negros em Portugal. Uma presença silenciosa, Lisboa, Caminho, 1988 ; Isabel Castro Henriques, A Herança Africana em Portugal, Lisboa, CTT, 2009.

38 J oão Nuno Coelho, Portugal. A Equipa de todos nós. Nacionalismo, futebol e media, Porto, Afrontamento, 2001.

39 Sérgio Campos de Matos, História, Mitologia, Imaginário Nacional. A História no Curso dos Liceus (1895-1939), Lisboa, Livros Horizonte, 1990.

40 J osé Manuel Sobral, Portugal..., op. cit.

41 Maria Isabel J oão, Memória e Império..., op. cit.
} 
Nuno Domingos, «Les reconfigurations de la mémoire du colonialisme portugais: récit et esthétisation de l'histoire », Histoire@Politique, [en ligne], n² 29, mai-août 2016, www.histoire-politique.fr

Fernando Garcia, journaliste à l'Emissora Nacional (la radio nationale), posa les éléments d'une chronologie officielle de sa vie qui, en grande mesure, a perduré jusqu'à la mort du joueur ${ }^{42}$. Selon cette autobiographie, Eusébio était un jeune qui avait grandi dans les quartiers pauvres et pittoresques de la banlieue de Lourenço Marques, avec leurs marchés colorés, leurs traditions locales, leurs habitants aux vêtements traditionnels. Il a été éduqué par une mère dévouée, Elisa. Son père, un Angolais métis, travailleur dans les chemins de fer, décéda alors qu'Eusébio n'était âgé que de 8 ans. Catholique, appliqué, bien éduqué, Eusébio révéla son talent lors des matchs disputés dans son quartier de Mafalala. Le déménagement vers le centre de la ville a marqué un tournant dans sa vie. Eusébio connaissait peu le centre de la ville car, selon l'argumentaire de sa biographie, il était très attaché à son quartier ${ }^{43}$. À 18 ans, il était déjà une idole locale. Il fit le grand saut vers Lisbonne, avec un transfert polémique à Benfica. Au-delà de ses grandes prouesses sportives, d'autres faits aident à tracer les caractéristiques de l'homme, notamment son incorporation médiatique au sein de l'armée portugaise, en 1963, et son mariage vertueux avec Flora Bruheim qui, elle aussi, venait du Mozambique. Cette union a été largement suivie dans les revues et décrite comme l'aboutissement romantique d'un grand amour. Eusébio était un homme de famille. Toujours selon ce récit, le succès n'a pas abîmé sa simplicité et son humilité. Ces qualités étaient celles qui, depuis longtemps, définissaient le comportement idéalisé de l'Africain assimilé. Plus globalement, ces qualités étaient celles de la classe ouvrière respectable, qui persistaient même lors des rares cas de mobilité sociale ascendante.

Les étapes de la vie d'Eusébio se déroulent dans un cadre social et historique idéalisé. Le récit de sa vie naturalise une définition « correcte» du quotidien. Selon cette biographie exemplaire, Eusébio n’a pas grandi dans les quartiers ségrégués de Lourenço Marques où l'on manquait de tout, où la malnutrition était courante, où se propageaient d'innombrables maladies et où le taux de natalité était sept fois supérieur à celui de la «ville de ciment » (le centre-ville). Eusébio ne faisait pas partie d'une communauté d'individus victimes d'une violence continue, écartés du centre de la ville, contrôlés et surveillés, exploités comme main-d'œuvre bon marché, obligés à émigrer en Afrique du Sud pour survivre. Eusébio n'a pas grandi dans une ville où lesdits indigènes ne possédaient pas les mêmes droits civils, juridiques et politiques que les blancs, où ils ne pouvaient pas fréquenter les mêmes espaces de loisir, les mêmes écoles, les mêmes hôpitaux, les mêmes moyens de transport que les Européens ${ }^{44}$.

Si cette représentation de l'empire, banalisée par la vie d'un joueur de football, a été construite à une période où la censure était très active, spécialement en ce qui concernait la construction d'images sur l'empire, ce récit exemplaire a survécu à l'empire. Après 1974, Eusébio est demeuré populaire, continuant à accompagner le Benfica et la sélection nationale. Il a appuyé certains partis et candidats politiques, presque toujours de droite, mais sa valeur en tant qu'atout électoral n’a pas été très

\footnotetext{
42 Eusébio da Silva Ferreira, O meu nome é Eusébio, Mem Martins, Europa-América, 1966.

43 Ibid.

44 Sur la situation des banlieues de Lourenço Marques au XX ${ }^{\mathrm{e}}$ siècle, voir J eanne Marie Penvenne, African Workers and colonial racism. Mozambican Strategies and Struggles in Lourenço Marques, 1877-1962, London, James Currey, 1995; António Rita-Ferreira, Os Africanos de Lourenço Marques, Lourenço Marques, Separata das Memórias do Instituto de Investigação Científica de Moçambique, 1967-1968 ; Valdemir Zamparoni, Entre Narros e Mulungos: colonialismo e paisagem social em Lourenço Marques, c.1890. c.1940, thèse de doctorat en histoire, Université de São Paulo, 1998.
} 
Nuno Domingos, «Les reconfigurations de la mémoire du colonialisme portugais: récit et esthétisation de l'histoire», Histoire@Politique, [en ligne], n² 29, mai-août 2016, www.histoire-politique.fr

probante. À de nombreuses reprises, il a affirmé que sa politique était le football ${ }^{45}$. Son désintérêt pour la politique a renforcé le consensus autour de sa personne, généré après sa mort. Son décès a produit un phénomène de commotion nationale et de manifestation populaire, particulièrement évident lors des funérailles épiques du joueur, un jour de fortes pluies. Cette ferveur s'est manifestée sous de nombreuses formes et a été peu à peu encadrée dans les discours des représentants du champ politique, des notables des partis, des commentateurs de la télévision, mais aussi des dirigeants sportifs. L'énorme couverture médiatique de sa mort a amplifié l'événement de manière extraordinaire et les audiences ont prouvé son importance nationale.

Le champ politique s'est très tôt montré désireux d'officialiser cette émotion nationale. Par le biais d'une décision unanime du parlement portugais, le corps d’Eusébio a été transféré au Panthéon national, lieu où reposent plusieurs héros nationaux ${ }^{46}$. Le texte officiel justifiant l'enterrement du sportif au Panthéon met en avant le fait que le joueur a été un « jalon dans la diffusion, la globalisation de l'image et de l'importance du Portugal dans le monde (...). Immédiatement reconnu partout dans le monde et rattaché à l'image du Portugal, grâce à sa personnalité, à son affabilité et à son humilité (...). Les succès sportifs, toutefois, n'impressionnent pas davantage que son côté humain : la gloire ne lui est jamais montée à la tête et tous ceux qui l'ont connu admiraient sa simplicité, son humilité et son sens de la solidarité. N'oublions pas les qualités humaines qui ont fait de lui une référence dans le partage et l'aide au prochain, ayant donné son visage et son image à diverses campagnes de solidarité, expression de son authentique et naturelle affabilité et son enthousiasme ».

La patrimonialisation étatique d'Eusébio et son élévation au rang de héros national étaient justifiées par des caractéristiques depuis longtemps mises en avant. L'exposition organisée ensuite par l'Assemblée de la République a rappelé par le biais de vidéos et de la memorabilia, toutes les étapes connues de sa biographie exemplaire. Plus de quarante ans après, les récits biographiques sur Eusébio, tant dans le registre officiel venu du champ politique que dans le registre médiatique, reproduisent presque tout ce qui avait été élaboré dans les années 1960, époque pendant laquelle le pays était gouverné par une dictature qui menait une guerre en Afrique pour la défense de son empire.

La reproduction d'une mémoire officielle de l'empire n'était pas le principal objectif de la patrimonialisation d'Eusébio. Maintenir intacte l'histoire coloniale était fondamentalement un élément indispensable pour la préservation de la figure du héros national. En 2015, le Portugal manque de héros consensuels. Dans un contexte de grave crise économique, l'éloge d'Eusébio a fondamentalement pour fonction de rendre naturel le cadre national et de renforcer le sentiment d'unité. Les discours qui ont accompagné la cérémonie au Panthéon soigneusement mise en scène ont insisté sur l'universalité d'Eusébio et son exemple humain, insistant sur son image

\footnotetext{
45 Gary Armstong, « The Migration of the Black Panther: an interview with Eusébio of Mozambique and Portugal », dans Gary Armstrong, Richard Giulianotti (eds.), Football in Africa, Conflict, Conciliation and Community, Hampshire, Palgrave, 2004, p. 247-268.

46 Réouvert en 1965 par l’Estado Novo, le panthéon national a reçu en 1966 Almeida Garrett, Guerra J unqueiro, J oão de Deus, Manuel de Arriaga, Óscar Carmona, Sidónio Pais et Teófilo Braga. Après le 25 avril, les corps de Humberto Delgado, Aquilino Ribeiro, Amália Rodrigues et Sophia de Mello Breyner Andresen y ont été placés. Le corps d’Eusébio a vu son enterrement au Panthéon confirmé par le projet de résolution, $\mathrm{n}^{\circ} 1232 / \mathrm{XII}$.
} 
Nuno Domingos, «Les reconfigurations de la mémoire du colonialisme portugais: récit et esthétisation de l'histoire», Histoire@Politique, [en ligne], n² 29, mai-août 2016, www.histoire-politique.fr

fédératrice auprès des partis politiques et des différentes idéologies. Le président de la République, Aníbal Cavaco Silva, répéta ce qui avait déjà été écrit dans les préfaces de plusieurs biographies d'Eusébio et qui, en grande partie était déjà mentionné dans le texte qui officialisait l'enterrement d'Eusébio au Panthéon ${ }^{47}$.

Les grands héros de la culture populaire, reconnus grâce à leur maestria pour des activités pratiquées par une grande part de la population et pas seulement au sein d'élites fermées, sont utiles pour renforcer la représentation d'une nation plastique, intégratrice, insensible aux changements ou aux projets de rupture, une nation dont le caractère national est défini par l'humilité et le sacrifice.

\section{L'histoire et le patrimoine comme récits de l'empire}

Les récits exemplaires légitimant la pastorale de l'empire portugais peuvent s'appuyer sur la vie d'un joueur de football comme Eusébio da Silva Ferreira. Ils peuvent aussi emprunter des voies plus spécialisées et symboliquement légitimes, notamment au sein de la recherche académique. Malgré de nouvelles études menées sur l'espace colonial portugais depuis le 25 avril 1974, surtout dans les deux dernières décennies, le champ scientifique, notamment l'histoire et les sciences sociales, peine toujours autant à ouvrir une discussion plus approfondie sur l'expérience coloniale portugaise. Les minces frontières entre le travail académique, la divulgation scientifique et le discours officiel du champ du pouvoir se perçoivent de manière particulière dans les thématiques liées à l'histoire nationale. Certains ouvrages espèrent mieux se vendre en reprenant les sentiments nationaux exprimés dans les anciens récits linéaires. La multiplication des récits sur le «fait national», souvent offerts par le genre biographique, n'a pas cessé avec le 25 avril 1974. Au contraire, ces récits ont représenté une dimension importante des stratégies de ventes des grands agents de la diffusion et du commerce culturels, comme le Círculo de Leitores (Cercle des Lecteurs) ${ }^{48}$, club du livre de la multinationale Bertelsmann, également connu pour promouvoir des ouvrages sur le Portugal, vendus en porte-à-porte, avec d'énormes tirages ${ }^{49}$. À la même période, la création de la Commission nationale pour la commémoration des découvertes portugaises (1986-2002) a renouvelé, au plus haut niveau, l'intérêt pour la célébration des « découvertes ». L'activité de la commission est source de discussions et de polémiques, notamment en ce qui concerne l'évaluation de l'héritage qu'elle laisse au champ scientifique, en comparaison avec son action menée dans le domaine commémoratif50. Une histoire politique, écrite de manière fluide, à statut commercial, débarrassée de la perturbation que causent les nombreuses notes de bas de page, la réflexion théorique et les séries statistiques, favorise le retour de modèles littéraires dramatisés, tournés désormais vers un plus large marché de lecteurs. Le désintérêt pour l'étude des sociétés coloniales, de ses

\footnotetext{
47 « Les heures de gloire ne lui sont pas montées à la tête et tous ceux qui connaissent Eusébio admirent sa simplicité, son bon coeur, son sens de la solidarité », préface du livre de J oão Malheiro, Eusébio. A biografia autorizada, Vila do Conde, Quidnovi, 2012, p. 10.

48 Note du traducteur : le Cercle des lecteurs correspond au Grand livre du mois français.

49 Inês Brasão, Nuno Domingos, «A História de um clube de livros », dans Diogo Ramada Curto (dir.), Sociologia da leitura em Portugal no século XX, Lisboa, Fundação Gulbenkian, 2006.

50 Diogo Ramada Curto, «A memória dos descobrimentos, da expansão e do império colonial », dans Bruno Monteiro, Nuno Domingos (eds), Este País não Existe, Porto, Deriva, 2014, p. 95.
} 
Nuno Domingos, «Les reconfigurations de la mémoire du colonialisme portugais: récit et esthétisation de l'histoire », Histoire@Politique, [en ligne], n² 29, mai-août 2016, www.histoire-politique.fr

groupes, de ses populations et de ses relations de pouvoir, favorise une étude focalisée sur la métropole, dans une représentation du processus historique établie par l'analyse des relations politiques et diplomatiques privilégiant l'action des élites et surtout des dirigeants ${ }^{51}$.

Le champ scientifique historique, en plein développement depuis le 25 avril 1974, n’a pas manqué de placer l'expérience de l'empire au cœur de ses thèmes de recherche et de ses problèmes, plusieurs d'entre eux étant déjà définis au cours de l'Estado Novo. L'empire continue à être un ample champ d'analyse, institutionnalisé dans des départements et des centres de recherche : anthropologie, études africaines, histoire, études culturelles, histoire de la science. Thème privilégié dans les logiques de consécration interne, l'empire a favorisé des stratégies d'internationalisation. Surtout tournées vers la consommation interne, plusieurs publications académiques ont appuyé une vision glorificatrice de l'empire. Certaines d'entre elles sont l'expression du travail développé dans des départements et centres de recherche, dans des universités publiques et privées.

L'étude du patrimoine a toujours été l'un des moyens dominants de représentation de l'imaginaire national. Pendant l'Estado Novo, l'évocation du patrimoine était directement historiciste (pour utiliser les termes communs employés dans le champ de l'architecture) et propagandiste, tout en étant liée au tourisme. De nos jours, si l'étude du patrimoine n'est ni historiciste ni propagandiste, elle ne constitue pas moins une des principales médiations mettant en évidence l'analyse des héritages matériels de l'empire ${ }^{52}$. Filtrée par des langages spécifiques, l'analyse du patrimoine finit plusieurs fois par réifier la chronologie, les thèmes et les problèmes de l'histoire nationale. Spécialistes et chercheurs se distinguent en appréhendant la réalité en termes nationaux : l'auteur portugais, le style portugais, l'école portugaise. Élément de consécration interne de champs d'activité, référent symbolique sur lequel repose la reproduction des structures institutionnelles, des pouvoirs et des opportunités professionnelles, l'empire n'est pas seulement une représentation. Pour les disciplines qui s'occupent du patrimoine, l'empire est un énorme espace d'opportunités. Ce qu'ont produit les Portugais dans les contextes impériaux est une matière d'analyse abondante ${ }^{53}$.

L'exploitation de cette fierté pour le patrimoine de l'empire s'est faite grâce à l'articulation entre la production académique et les moyens de communication de masse. Certaines émissions de télévision de prime time ont demandé aux téléspectateurs de voter pour les « merveilles » que les Portugais ont laissées à travers le monde, merveilles choisies par un ensemble de professeurs et de chercheurs ${ }^{54}$. Un groupe de spécialistes a protesté contre cette sélection, qui faisait la part belle aux bâtiments militaires et aux églises, et qui s'affranchissait des contextualisations

\footnotetext{
${ }^{51}$ Nous n'apprendrions pas grand-chose sur la société dans laquelle Eusébio da Silva Ferreira a grandi en lisant l'Histoire du Portugal, coordonnée par Rui Ramos. Vendue par un éditeur commercial puis publiée en fascicules avec un hebdomadaire à grand tirage, cet ouvrage a recours à certains vieux processus de l'historiographie de divulgation pour transmettre un ensemble d'idées. Voir Rui Ramos (coord.), História de Portugal, Lisboa, Esfera dos Livros, 2009.

52 L'État s'est préoccupé depuis longtemps des questions du patrimoine, éditant différentes publications spécialisées, réalisant des documentaires et des films d'actualités.

53 L'édition du Patrimoine portugais du monde, coordonnée par J osé Mattoso, un important inventaire du bâti laissé dans les anciennes possessions de l'empire, est un exemple, en dépit du souci de mise en contexte historique, de ce regard patrimonialiste sur le passé: José Mattoso (dir.), Património de Origem Portuguesa no Mundo, Lisboa, Fundação Gulbenkian, 2012.

54 Dirigés par l'historien Pedro Dias de l'université de Coimbra.
} 
Nuno Domingos, «Les reconfigurations de la mémoire du colonialisme portugais: récit et esthétisation de l'histoire », Histoire@Politique, [en ligne], n² 29, mai-août 2016, www.histoire-politique.fr

historiques nécessaires pour comprendre les fonctions de ces espaces dans le cadre du projet colonial portugais. Sur un modèle similaire, une précédente émission de télévision avait proposé une liste des Portugais les plus importants de l’histoire ( « les grands Portugais »). Dans cette liste, dont la première place est revenue à Salazar, on trouvait de nombreux noms liés à la geste impériale.

Ce nationalisme patrimonialiste se retrouve dans de récentes publications sur les héritages de l'architecture coloniale portugaise en Afrique au cours du XX ${ }^{\mathrm{e}}$ siècle. Cet exemple permet d'observer de quelle façon l'histoire interne produite par des champs d'activité spécifiques est perméable aux récits qui constituent les piliers de la grande histoire, aidant à les reproduire et à les naturaliser.

\section{Esthétiser l’empire}

$\mathrm{Au}$ cours du colonialisme tardif, un groupe d'architectes et d'urbanistes portugais a laissé en Afrique d'importants travaux. En dépit des recherches et des publications pionnières de José Manuel Fernandes, qui ont essayé de mettre en avant leur signification ${ }^{55}$, cette ouvre est restée peu connue au Portugal. Si l'histoire de l'architecture portugaise avait marginalisé ces créateurs, leur récupération a obligé à repenser l'histoire même de la discipline ${ }^{56}$. Comment mettre en rapport le travail d'architectes modernistes avec un régime où les droits politiques et syndicaux n'existaient pas et où les mouvements et les associations étaient surveillés ? Comment raconter cette histoire en préservant le récit du modernisme architectural du Portugal, déjà dominant avant la fin del'Estado Novo ?

Imposant à l'histoire une dynamique narrative où prédominent les auteurs, les œuvres, les innovations techniques et esthétiques, les analyses de l'architecture coloniale cherchent à interpréter la relation de cette dynamique avec le cadre politique ambiant. La construction moderniste en Afrique s'inscrivait dans la catégorie de l'architecture tropicale ${ }^{57}$. Justifié par le besoin des architectes qui devaient adapter leurs œuvres au climat des tropiques - ce qui suggérait une nouvelle variante du modernisme-, l'usage de l'expression tropicale était propice à l'euphémisation historique. Les travaux sur le modernisme portugais dans les colonies ont renforcé l'existence d'une architecture luso-africaine, de matrice portugaise, dans le cadre d'une portugalité tropicale ${ }^{58}$. La tentative d'articuler le contexte politique avec une analyse centrée sur les présupposés techniques et stylistiques, qui ont lié l'avant-garde nationale au mouvement moderne international et à ses plus notables représentants, a conduit à diverses lignes d'argumentation. Séparant l'architecture de l'histoire, on créait l'idée d'une autonomie professionnelle,

\footnotetext{
55 J osé Manuel Fernandes, Geração Africana. Arquitectura e Cidades em Angola e Moçambique, 19251975, Lisboa, Livros Horizonte, 2002.

56 Dans l'entrée « architecture » présente dans le dictionnaire de l'histoire du Portugal, il n'y a aucune référence à l'espace colonial. Voir Nuno Portas, « Arquitectura », dans António Barreto, Maria Filomena Mónica (eds.), Dicionário de História de Portugal, Porto, Figueirinhas, 1999, p. 118-126.

57 Un de ses classiques était Maxwell Frey, J ane Drew, Tropical Architecture in the Humid Zone, New York, Reinhold, 1956.

58 José Manuel Fernandes, «Arquitectura e urbanisno no espaço ultramarino português », dans Francisco Bethencourt, Kirti Chaudhuri (eds.) História da Expansão Portuguesa, vol. 5, op. cit. ; J osé Manuel Fernandes, Arquitectura e Urbanismo de Matriz Portuguesa, Lisboa, Caleidoscópio/Universidade Autónoma, 2011; Ana Magalhães, Inês Gonçalves, Moderno Tropical. Arquitectura em Angola e Moçambique, Lisboa, Tinta da China, 2009 ; Ana Vaz Milheiro, Nos Trópicos sem Le Corbusier, Arquitectura Luso-africana no Estado Novo, Lisboa, Relógio D’Água, 2012.
} 
Nuno Domingos, «Les reconfigurations de la mémoire du colonialisme portugais: récit et esthétisation de l'histoire», Histoire@Politique, [en ligne], n² 29, mai-août 2016, www.histoire-politique.fr

conquise dans le cadre de l’Estado Novo, tant en métropole que dans l'empire. En lien avec cette thèse, il était suggéré que cette autonomie avait été conquise dans le cadre d'une résistance politique à l'Estado Novo, avec des conséquences personnelles reconnues pour certains architectes appartenant à l'opposition au régimes9. Enfin, certains travaux récents, modifiant les termes de la discussion, défendent que le régime s'est transformé à cette époque, devenant plus progressiste. Cela était visible sur le terrain colonial où l'État a initié un ensemble de projets de construction d'infrastructures ${ }^{60}$.

Ces interprétations, qui répondent aux sollicitations analytiques et aux modèles de consécration d'un champ académique international spécifique, marginalisent le fait que cet espace de production d'auteurs et d'œuvres était aussi un univers où de nombreux professionnels dépendaient de la demande de l'État colonial, à un certain moment plus préoccupé de l'efficacité des travaux que du style, pour développer une carrière et survivre. Mais le point fondamental pour comprendre le rapport entre la demande étatique et l'offre professionnelle exige un questionnement sur les fonctions de ce patrimoine bâti dans le cadre d'un système de domination coloniale ${ }^{61}$. Dans les années 1960, stimulés par des plans de mise en valeur économique étatiques et par une augmentation de l'investissement privé, des grands investissements en infrastructure, pour lesquels la contribution des architectes et urbanistes était fondamentale, ont été réalisés ${ }^{62}$.

Au-delà du discours formel qui habite l'histoire de ce champ d'activité, le bâti impérial raconte d'autres histoires, qui ont été passées sous silence. Les plus marquantes sont celles qui nous présentent une histoire urbaine coloniale appuyée sur la production de plans qui, jusqu'à très tardivement, se sont fondés sur des modèles ségrégationnistes. À ce sujet, les idées de l'architecte J oão Aguiar sont emblématiques. João Aguiar fut l'un des principaux théoriciens du tropicalisme portugais, responsable du cabinet d'urbanisation coloniale (1944-1951, qui devient, à partir de 1951, le cabinet d'urbanisation d'Outre-mer) et l'auteur de nombreux plans d'urbanisation de villes africaines. Ses idées révèlent précisément que les options stylistiques et techniques d'urbanistes et d'architectes se trouvent mises au service de fonctions spécifiques: créer des centres urbains modernes et fonctionnels, où l'habitation traduit la hiérarchie sociale, professionnelle et raciale, où la localisation des populations est au service de la fonctionnalité économique et des besoins en main-d'œuvre, où les équipements sociaux cherchent à stabiliser ces populations ${ }^{63}$. $\mathrm{Si}$ dans les années 1960, le discours racialiste disparaît des plans urbanistiques et des rhétoriques du régime, les mêmes fonctions modernes, qui cherchent à rendre efficaces les équipements de l'État et l'activité économique, fabriquant un

\footnotetext{
${ }^{59}$ Interprétation présente surtout dans l'œuvre de J osé Manuel Fernandes. Voir J osé Manuel Fernandes, A Geração Africana..., op. cit.

60 C'est l'argument avancé par Ana Vaz Milheiro : Ana Vaz Milheiro, Nos Trópicos sem Le Corbusier..., op. cit.

61 A. R. Ferreira, Obras públicas em Moçambique: Inventário da produção arquitectónica executada entre 1933 e 1961, mémoire de maîtrise, département d'architecture, Faculté de sciences et technologies, université de Coimbra, 2006.

62 Victor Pereira, « A economia do Império e os Planos de Fomento », dans Miguel Bandeira J erónimo (dir.), O Império Colonial em Questão, Lisboa, Edições 70, 2011, p. 251-285.

63 J oão António de Aguiar, L’habitation dans les pays tropicaux, Lisboa, Fédération internationale de l'habitation et de l'urbanisme, 1952.
} 
Nuno Domingos, «Les reconfigurations de la mémoire du colonialisme portugais: récit et esthétisation de l'histoire », Histoire@Politique, [en ligne], n² 29, mai-août 2016, www.histoire-politique.fr

encadrement des colons et des autochtones, continuent à déterminer le travail des urbanistes et des architectes, à une époque où la guerre coloniale est généralisée ${ }^{64}$.

Les champs qui traitent de l'excellence esthétique, même quand ils font l'éloge des styles internationaux, placent ces styles dans la perspective de l'appartenance nationale, observant un patrimoine qu'il s'agit d'étudier pour sa valeur artistique plus que pour ses fonctions pratiques, historiquement significatives. Aujourd'hui, certaines analyses du legs architectural portugais dans le monde s'insèrent dans ce processus plus large de patrimonialisation de l'héritage national et impérial, sujet de projets d'études et de publications. Cette représentation architecturale de l'espace colonial reproduit de manière spécifique la mémoire officielle de l'empire, se projetant dans un marché qui s'appuie sur la naturalisation de la vision du passé. N'étant pas le reflet direct d'une idéologie qui lui est imposée, comme si elle provenait d'une politique décrétée par l'État, cette représentation se manifeste dans les luttes internes du champ, s'imposant comme un mécanisme des distinctions en jeu, et un mécanisme fondamental de consécration professionnelle.

\section{Qui construit l'image de l'empire ?}

Le cas du joueur de football né au Mozambique et celui de l'analyse de l'architecture coloniale moderne révèlent comment, depuis le 25 avril 1974, des manières spécifiques de raconter l'histoire nationale continuent à reproduire les représentations dominantes de l'empire portugais, perpétuant une même grille de lecture. Cette construction de l'empire comme catégorie de connaissance et d'interprétation du monde, tend à confirmer et à conférer une légitimité à des images du passé qui, même après la fin de l'empire, sont encore des éléments fondamentaux de la subjectivation de l'identité nationale. Le discours dominant continue à affirmer que même s'il n'a plus d'empire, le Portugal a laissé une culture à travers le monde, prolongeant une globalisation multiculturelle, intégratrice, métissée qui a pris la forme d'une expérience, d'un échange. Comme nous l'avons vu dans la construction biographique d'Eusébio ou dans la représentation de l'héritage architectural colonial, les processus de conquête et de domination coloniale sont effacés ${ }^{65}$. La mémoire de l'expansion est aujourd'hui une pièce fondamentale d'un «terroir portugais ». La «geste des découvertes » confirme l'exceptionnalité nationale et elle est commercialisée à côté de produits nationaux, comme les mets et les vins vendus aux visiteurs étrangers et aux citoyens portugais ${ }^{66}$.

Les processus de narration de cette mémoire coloniale ont néanmoins été renouvelés, notamment après les indépendances africaines. De nouvelles dynamiques

64 Mário de Oliveira, Problemas Essenciais do Urbanismo no Ultramar. Estruturas urbanas de integração e conveniência, Lisboa, Agência-Geral do Ultramar, 1962.

65 Ces facultés nationales sont ensuite utiles pour la promotion urbaine de villes comme Lisbonne, où le multiculturalisme semble renforcer les logiques de reconfiguration sociale, notamment la gentrification de certains quartiers.

66 Dans une réflexion récente, Elsa Peralta a étudié la constante re-création du quartier lisboète de Belém en tant que complexe mnémonique. Ici, la dynamique institutionnelle autour de la mémoire officielle - à laquelle va probablement s'ajouter la construction d'un musée des Découvertes, soutenue par la mairie - se conjugue aujourd'hui avec une puissante marchandisation touristique. Cet aspect, déjà évoqué dans un texte de Francisco Bethencourt, prend une énorme importance. L'exposition universelle de 1998, dans la zone orientale de Lisbonne, où a également été construit un pont appelé Vasco da Gama, dédiée au thème très politiquement correct des océans, a renforcé cette vocation mémorialistique des rives de Lisbonne. 
Nuno Domingos, «Les reconfigurations de la mémoire du colonialisme portugais: récit et esthétisation de l'histoire», Histoire@Politique, [en ligne], n² 29, mai-août 2016, www.histoire-politique.fr

institutionnelles reconfigurent la mémoire de l'empire promouvant l'histoire des individus et des patrimoines qui prennent l'empire comme une donnée acquise, et reproduisant ainsi un imaginaire qui demeure un pilier fondamental du nationalisme portugais. La répétition continue d'un récit exemplaire de la mémoire de l'empire dépend aussi de l'histoire et de l'organisation institutionnelle contemporaine de champs d'activité spécifiques. La reproduction de représentations du passé impérial constitue une forme indirecte de capital symbolique à l'intérieur de ces champs. C'est le cas lorsqu'on célèbre les victoires sportives d'un joueur africain, victoires qui satisfont les intérêts de nombreux agents : des dirigeants de clubs, d'associations ou de fédérations sportives, des journalistes et même des supporters. C'est le cas également dans le cas de l'histoire de l'architecture coloniale avec les perspectives disciplinaires, les curriculums universitaires ou les biographies artistiques. De cette manière, la mémoire de l'empire n'est pas seulement une représentation, elle continue de remplir des fonctions pratiques.

\section{Traduit par Victor Pereira}

\section{L'auteur}

Nuno Domingos est chercheur de l'Institut de sciences sociales de l'université de Lisbonne. Il a travaillé sur le colonialisme portugais au Mozambique durant le $\mathrm{XX}^{\mathrm{e}}$ siècle et sur l'Estado Novo à partir de l'analyse des pratiques et des consommations culturelles. Sur ces thèmes, il a récemment publié "Colonial Architectures, Urban Planning and the representation of Portuguese Imperial History" (J ournal Of Portuguese Social Sciences, vol. 14, n.0ㅜㅇ, pp. 235-255) ; "The malicious football game: urban interactions and power relations in Lourenço Marques, capital of colonial Mozambique (Journal of Southern African Studies, vol. 41, Issue 2, April 2015, pp. 315-334).

\section{Résumé}

L'empire colonial est un élément central de la construction officielle de l'identité nationale portugaise. Le processus d'indépendance des anciennes colonies après le 25 avril 1974 a conduit à la reconfiguration des manières de raconter le passé. Basé sur deux études de cas, cet article prétend analyser comment des nouvelles médiations et des nouveaux récits ont été utilisés pour reconfigurer cette mémoire. Le cas de la construction biographique du joueur de football mozambicain Eusébio da Silva Ferreira, le grand héros du football portugais, et l'analyse de l'héritage de l'architecture coloniale moderne portugaise visent à démontrer le rôle que ces récits produits dans des champs d'activités spécifiques ont joué dans le maintien d'un discours portant sur la grandeur coloniale, discours qui est lui-même une dimension de l'institutionnalisation de ces champs.

Mots clés : colonialisme portugais ; identité nationale ; champs ; récits ; mémoire.

\section{Abstract}

"The Reconfiguration of the Memory of Portuguese Colonialism: Narrative and the Aestheticization of History"

The colonial empire is a central component of the official version of Portuguese national identity. In the aftermath of 25 April 1974, the independence process in the country's former colonies led to a reconfiguration of the manner in which the past is 
Nuno Domingos, «Les reconfigurations de la mémoire du colonialisme portugais: récit et esthétisation de l’histoire», Histoire@Politique, [en ligne], n² 29, mai-août 2016, www.histoire-politique.fr

discussed. This article seeks to examine how new mediations and narratives were used to reconfigure this memory. To that end, it draws upon two case studies: the biographical construction of Eusébio da Silva Ferreira, a Mozambican football player and national hero; the heritage of modern Portuguese colonial architecture. The author seeks to show how these narratives, which were produced in specific fields of activity, played a role in preserving a discourse of colonial grandeur - itself a reflection of the institutionalization of these fields.

Key words : Portuguese Colonialism; National Identity; Fields; Narratives; Memory.

Pour citer cet article : Nuno Domingos, «Les reconfigurations de la mémoire du colonialisme portugais : récit et esthétisation de l'histoire », Histoire@Politique, [en ligne], $n^{\circ}$ 29, mai-août 2016, www.histoire-politique.fr 